\title{
3D PRINTING OF FIBER-REINFORCED SOFT COMPOSITES: PROCESS STUDY AND MATERIAL CHARACTERIZATION
}

\author{
ClaysonC. Spackman \\ Graduate Research \\ Assistant \\ spackc@rpi.edu
}

\author{
Christopher R. Frank \\ Graduate Research \\ Assistant \\ frankc7@rpi.edu
}

\author{
Kyle C. Picha \\ Graduate Research \\ Assistant \\ pichak@rpi.edu
}

\author{
Johnson Samuel
Assistant Professor \\ Johnson Samuel
Assistant Professor \\ samuej2@rpi.edu
}

\author{
Department of Mechanical Aerospace and Nuclear Engineering \\ Rensselaer Polytechnic Institute, $1108^{\text {th }}$ Street, Troy, NY 12180, USA
}

\begin{abstract}
Fiber-reinforced soft composites (FrSCs) are composites that are made up of polymeric fibers with specific material properties and hierarchical length-scales, embedded within another soft-polymer matrix. This paper is aimed at systematically studying the effect of key processing parameters viz., fiber mat alignment, area coverage, and surface energy of the fiber carrier substrate, on the tensile properties and failure mechanisms seen in 3D printed FrSCs. A novel electrospinning-based“direct-write” system isused for creating the aligned and random nylon fiber mats. The fiber mats are then characterized for their diameter distributions, effective area coverage, number density, and tensile properties. The surface energy of the fiber carrier substrate is found to be critical to the fiber transfer efficiency of the stamping operation used in the 3D printing process, with polytetrafluoroethylene-coated aluminum films being more effective due to their low surface energy. Tensile testing results show that depending on the extent of alignment and the fiber content present in the 3D printed composite, it can have a 40\%-260\% improvement in the elastic modulus over that of the base UV-curable polymer. The composites alsoshow evidence of characteristic failure mechanisms seen in the domain of nanocomposite materials, viz., fiber-induced local plastic deformation (crazing), crack arrest and deflection, fiber strengthening, and fiber pull-out. The evidence of fiber pull-out also points to the formation of an interfacial polymer sheath around the fibers. The elastic modulus of this sheath is estimated to be an order of magnitude higher than the base polymer.
\end{abstract}

Keywords: Fiber-reinforced soft composites, Electrospinning, Additive manufacturing, 3D printing

\section{INTRODUCTION}

Historically, fiber-reinforced polymer composites have been thought of as high-strength, light-weight materials that are composed of strong, load-carrying fibers (e.g. carbon/glass fibers, 5-6 $\mu \mathrm{m}$ in diameter and tens of centimeters in length) held together by an epoxy matrix. However, there is a growing class of applications involving fiber-reinforced soft composites (FrSCs) that has recently attracted significant scientific attention [1-5]. FrSCs are composites that are made up of polymeric fibers with specific material properties and hierarchical length-scales, embedded within another soft-polymer matrix. The emerging applications of FrSCs include 4D printing, biomimetic composites, and embedded sensing/actuation [1-5].These applications have highlighted the impending need to combine 3D printing technologies with the directed deposition of polymeric fiber networks.

Conventional polymer-based additive manufacturing technologies such as fused-deposition modeling [6], stereolithography [7] and inkjet-based printing of ultraviolet (UV) curable polymers [8] have been shown to combine multiple polymers into a single part. However, their applicability to the 3D printing of FrSCs is limited. While nanofiller-based inks or polymers can be used in the above processes, they do not provide control on the positioning or patterning of these fibrous structures, which is critical for applications requiring the use of laminated FrSCs, tunable composite structures and fiber-alignment enabled property enhancements.

Recently, Spackman et al. [9] demonstrated a hybrid, multi-material 3D printing process for fabricating laminated FrSCs that have characteristic length-scales in the tens of millimeters range. The printing process combined the conventional inkjet-based printing of UV curable polymers with the directed deposition of either aligned or random nylon fiber mats, in between each printed layer. The rolls of fibers were first generated using a far-field solution electrospinning process. These fibers were then transferred to the part being manufactured using a stamping operation. While this process was shown to be capable of printing multi-material 3D parts, there is little understanding of the effect of key processing parameters on the resulting material properties of the laminated FrSCs.

This paper builds on the prior work of Spackman et al. [9] and is aimed at systematically studying the effect of key processing parameters including fiber mat alignment, area coverage, and surface energy of the fiber carrier substrate on the tensile properties and failure mechanisms seen in 3D printed FrSCs. First, a near-field electrospinning process is developed to control the alignment and number density of the nylon fibers. A fiber transfer efficiency study is then conducted to determine the operational regime of the 3D printer. The resulting FrSC laminates are then characterized for their tensile properties and failure mechanisms.

The remainder of this paper is organized as follows. Section 2 presents a brief review of the 3D printing platform developed by Spackman et al. [9]. Section 3 presents the overall manufacturing process cycle for the 3D printing of FrSCs. Sections 4 and 5 present

${ }^{1}$ Corresponding author email: samuej2@rpi.edu

(C) 2016. This manuscript version is made available under the Elsevier user license

http://www.elsevier.com/open-access/userlicense/1.0/ 
the results from the electrospinning experiments and fiber transfer efficiency study, respectively. Section 6 presents the mechanical property characterization of 3D printed FrSCs followed by Section 7, which discusses the different failure mechanisms at play in these materials. Finally, Section 8 presents the specific conclusions that can be drawn from this study.

\section{OVERVIEW OF NOVEL 3D PRINTING PLATFORM}

The experiments presented in this paper were performed on the five-axis 3D printing platform designed by Spackman et al. [9] for the 3D printing of laminated FrSCs. This section will briefly review the capabilities of this printing platform for the purpose of continuity.

As shown in Fig. 1, the Spackman et al. [9]3D printing platform consists of the following key components: 1) a multi-nozzle inkjet-based polymer dispensing unit (item $a$ in Fig. 1) that is used to deposit the UV-curable polymer; 2) a fiber layering system (FLS) that stamps the fiber mats onto the part being printed (item $b$ in Fig. 1); 3) a surface preparation system comprising of a UV source (item $c$ in Fig. 1), rod lens (item $d$ in Fig. 1), and a heat lamp (item $e$ in Fig. 1); and 4) the substrate mount (item $g$ in Fig. 1). The system consists of an Aerotech ${ }^{\mathrm{TM}}$ five-axis linear motion system with the substrate being moved by Axes 1-2, and Axis 3 carrying the inkjet print-heads. Axes 4 and 5 provide motion for the FLS. Axes 1-2 have a positional accuracy of $\pm 100 \mathrm{~nm}$. The remaining axes have a positional accuracy of $\pm 1 \mu \mathrm{m}$. The UV source (Dymax ${ }^{\mathrm{TM}}$ BlueWave 200) and heat lamp (200 Watt) are mounted on pneumatic slides (item fin Fig. 1) fixed to the granite base of the 3D printer. A kinematic-coupling based pallet system is used to hold the substrate during the printing process. The printer has a build volume of $100 \mathrm{~mm} \mathrm{X} 100 \mathrm{~mm}$ X $50 \mathrm{~mm}$.

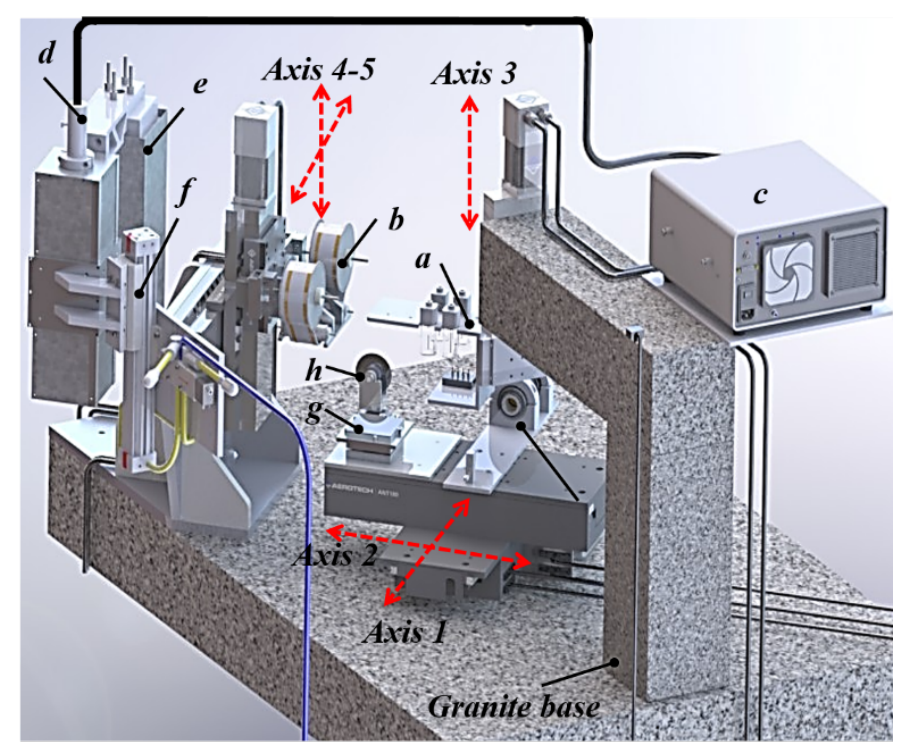

Fig. 1: 3D printing platform for fiber-reinforced soft composites ( $a$ - inkjet nozzles, $b$ - fiber layering system, $c$ - UV cure lamp, $d$ - UV curing rod lens, $e$ - heat lamp, $f$ pneumatic slides, $g$ - printing substrate, $h$ - tungsten carbide cutter) [9]

Multi-nozzle Inkjet-based Polymer Dispensing Unit: UV curable polymer inks that constitute the matrix of the composites are dispensed via an inkjet-based drop-on-demand system from MicroFab Technologies Inc. The system comprises of four piezoelectric micro-nozzles (MicroFab ${ }^{\mathrm{TM}}$ MJ-ABL series) with individual ink reservoirs, a pneumatic controller (MicroFab ${ }^{\mathrm{TM}}$ CT-PT4) and JetDrive III drive electronics to trigger each of the nozzles. The presence of multiple nozzles not only allows for printing multimaterial parts but it also enables the selection of different nozzle diameters in the 20-120 $\mu \mathrm{m}$ range.

Fiber Layering System (FLS): The FLS is used to transfer fiber mats onto the 3D printed part. The stamping region of the FLS is $50 \mathrm{~mm} \mathrm{X} 100 \mathrm{~mm}$ and is made of a high-density elastomer that provides the stamping force for depositing the fibers. A circular tungsten carbide cutter (item $h$ in Fig. 1) mounted on Axes 1 and 2 is used to cut the fiber mats into the required 2D geometry for each layer. The mount for the cutter is fixed to a servo motor that allows for the cutter to rotate in 90 degree increments in order to cut rectangular 2D patterns.

Additional design and performance metrics pertaining to this five-axis 3D printing platform can be found in Spackman et al. [9].

\section{MANUFACTURING PROCESS CYCLE FOR 3D PRINTING FIBER-REINFORCED SOFT COMPOSITES}

Figure 2 depicts the overall manufacturing process cycle used in this study for 3D printing fiber-reinforced soft composites. The process is comprised of the following steps:

- Step 1: Using electrospinning-based "direct-writing” techniques to manufacture polymer fiber mats on appropriate carrier substrates. The fiber mats are subsequently transferred to the FLS attached to the 3D printing platform mentioned in Section 2.

- Step 2: Inkjet printing and UV curing a layer of the polymer matrix. 
- Step 3: Cutting the fiber mat as per the 2D pattern required for the polymer layer printed in Step 2.

- $\quad$ Step 4: Stamping the cut fiber mat pattern onto the polymer layer printed in Step 2.

- $\quad$ Step 5: Repeating Steps 1-4 until the part is complete.

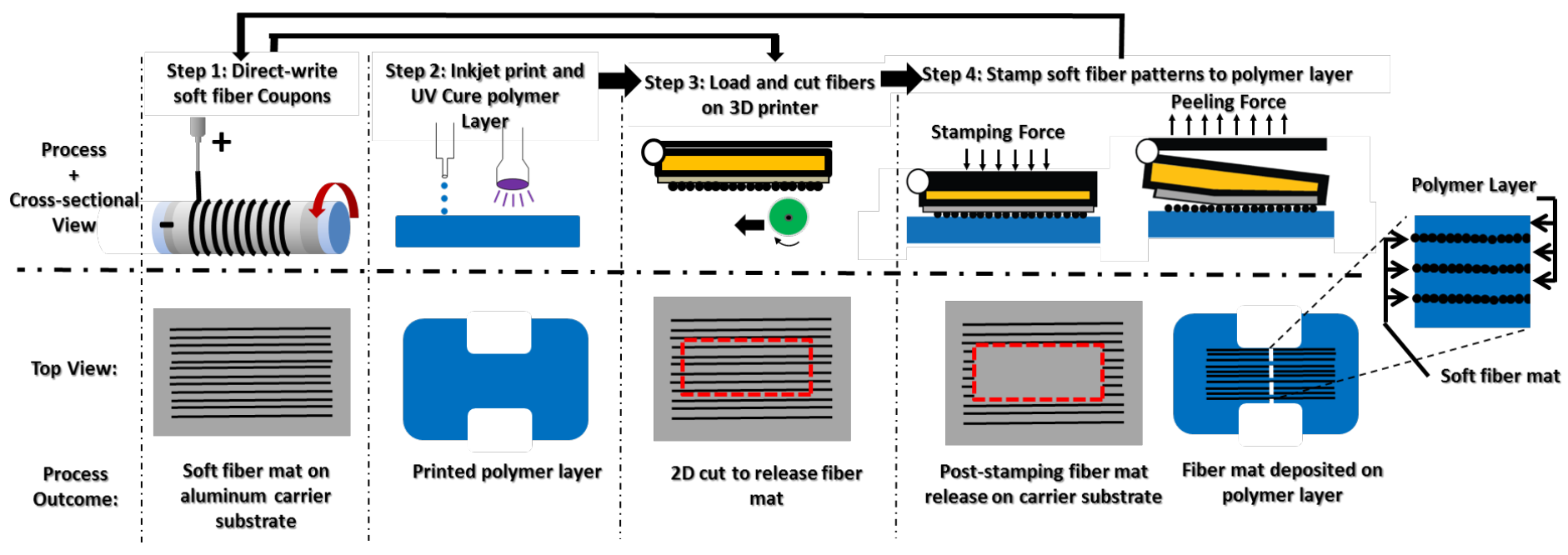

Fig. 2: Overall manufacturing process cycle for 3D printing FrSCs

The resulting FrSC part has a laminated structure with alternating layers of fiber mats and UV curable polymer (Fig. 2). While this processing technique is based on the 3D printing process created by Spackman et al. [9], there are three notable changes made in this study. These include:

1. Direct-writing of polymer fiber coupons. The fiber generation process reported by Spackman et al. [9] was based on the farfield solution electrospinning process and it relied on the design of parallel electrodes to manufacture rolls of aligned fiber mats. While that process was effective in fabricating large-area mats, it did not allow precise control over the fiber diameter, area coverage, and alignment/patterning, which is critical in studying the effect of processing parameters on the material properties of the 3D printed part. Therefore, a new electrospinning-based "direct-write" system was designed that operates both in the nearfield and far-field electrospinning modes while using a rotating drum collector. This study also focuses on characterizing the diameter distributions and the area coverage of the various fiber mats.

2. Carrier substrate. Aluminum carrier substrates of different surface energies were investigated here to study their impact on the efficiency of depositing the fibers during the stamping operation.

3. Modified fiber layering system. In the Spackman et al. [9] study, the fiber stamping operation was done using a fiber layering system (FLS) where the fiber carrier substrate was always parallel to the surface being printed. Subsequent 3D printing trials revealed that such a fiber layering system did not efficiently release the fibers from the carrier substrate, since it did not enable a peeling action to separate the fibers from the carrier substrate. To ensure an effective peeling action of the carrier substrate from the fibers, a spring-loaded hinge was incorporated into the FLS. This allowed for the fiber carrier substrate to be inclined at $30^{\circ}$ to the substrate surface (Fig. 2).

\section{FIBER MAT MANUFACTURING TECHNIQUE}

\subsection{Direct-writing of polymer fiber mats}

In order to achieve control over the spacing and patterning of the fibers, the near-field (NF) electrospinning process was selected for manufacturing the fiber mats [10]. This process is a variation of the near-field electrohydrodynamic jet printing process (E-jet) used for printing sub-micron droplets [11]. The solution used for the NF electrospinning consists of the polymer-of-interest dissolved in a solvent. A high voltage is then applied to the solution-carrying syringe and substrate combination. This leads first to the deformation of the liquid meniscus to form the Taylor cone [12]. Subsequently, the instability of the Taylor cone results in the formation of a continuous jet that could be used to direct-write continuous patterns. Once the jet is deposited on the substrate, the solvent evaporates over time and leaves behind the polymer fibers.

The accuracy of a NF electrospinning direct-write process is critically dependent upon the substrate moving at a speed that matches the speed of the polymer jet [12,13]. Common linear-motion platforms are not capable of matching the jet-ejection speeds encountered in NF electrospinning. Therefore, a custom-designed, rotating drum-collector was used to manufacture the fiber mats (Fig. 3a). The drum collector was a hollow polycarbonate (PC) tube (1.5”diameter, 0.25 ” thick), driven via a friction-fit belt. The variable speed motor (10-6000 RPM) driving the drum was separated from the high-voltage direct-write region by a 0.5” thick PC plate to avoid interference with the electric field used for the electrospinning process. 
The direct-write region of the drum collector had a composite cross-section consisting of a non-conducting polycarbonate drum coated with a quick-release, $100 \mu \mathrm{m}$ thick aluminum film (Fig. 3b). The quick-release, aluminum thin-film served both as the carrier substrate for the fibers and also as an electrode/grounding junction. In order to focus the electric field during the direct-writing process, a pin electrode (3 mm diameter) was also provided through the hollow PC tube (Fig. 3b). This additional electrode provided a tighter control on the direct-write patterns and also protected the bearings and the electric motor (being used to rotate the drum), from possible damage under the high-voltage conditions. Both the syringe pump and the drum collector were mounted on a three-axis precision motion platform with each axes having a $\pm 1 \mu \mathrm{m}$ positional accuracy and a $100 \mathrm{~mm}$ travel (Fig. 3a). The micro-scale positional accuracy of the platform combined with the focusing of the electric fields using the pin electrode enabled the direct-writing of fiber mats on the drum collector.

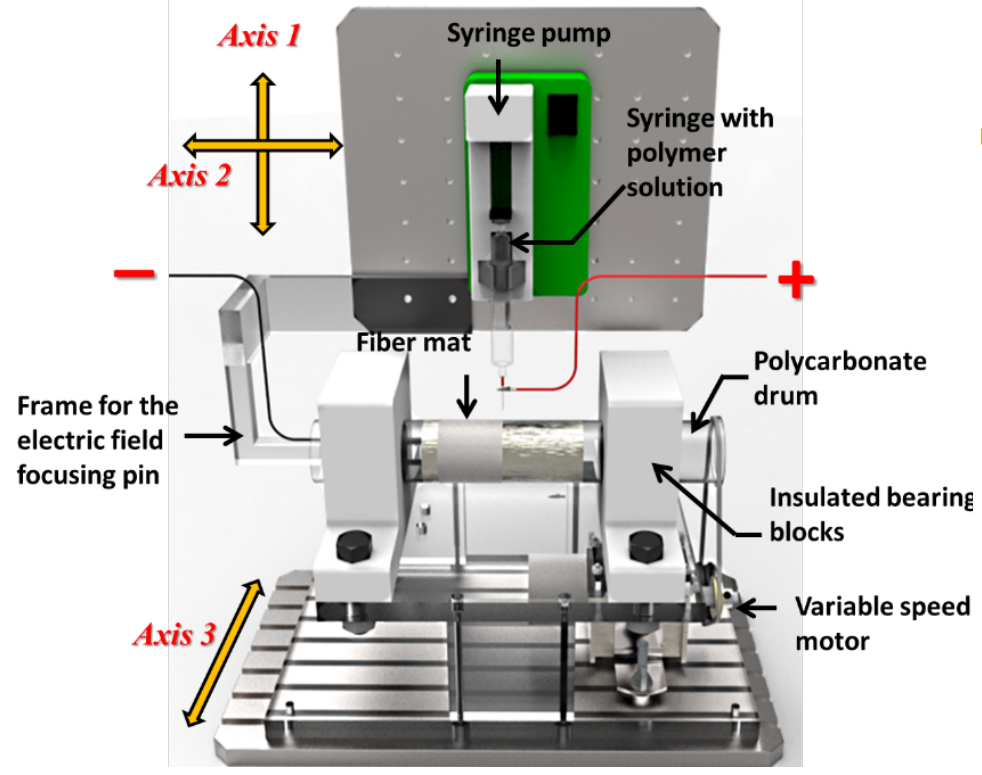

(a)

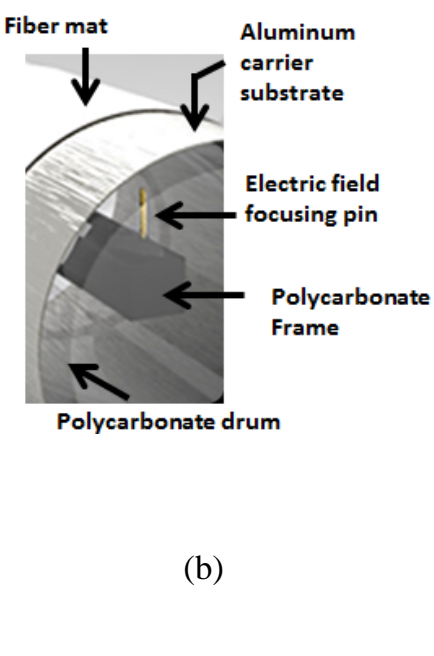

Fiber mat

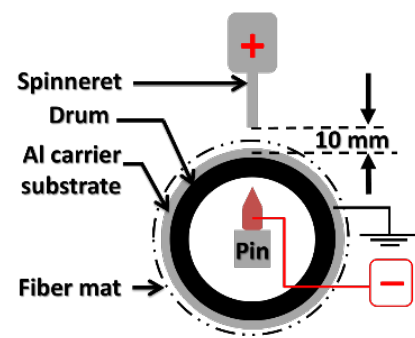

(c)

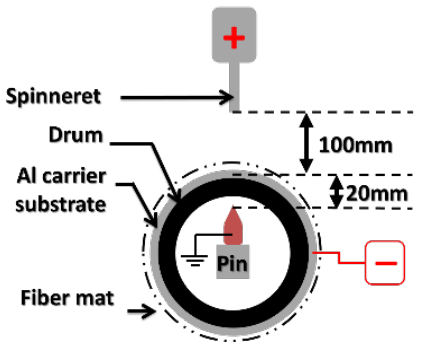

(d)

Fig. 3: Direct-write setup for manufacturing polymer fiber mats (a) Overall set-up configuration (b) Close up of the interior of the drum collector (c) Near-field configuration for aligned fiber mats (d) Far-field configuration for random fiber mats

\subsection{Fiber Mat Production Results}

Nylon-6 fibers were chosen as the reinforcement phase in this study [9,14]. Nylon 6 pellets (3 mm diameter) and formic acid (reagent grade $>95 \%$ to be used as solvent), were purchased from Sigma-Aldrich ${ }^{\mathrm{TM}}$ and used as received. A polymer solution concentration of $22.5 \mathrm{wt} \%$ was fed from the $30 \mathrm{~mL}$ capacity glass syringe (Multi-Fit ${ }^{\mathrm{TM}}$ ) fitted with a 27 gauge blunt-ended needle. A constant flow rate of $0.2 \mathrm{~mL} / \mathrm{hr}$ was controlled with a syringe pump (Razel ${ }^{\mathrm{TM}} \mathrm{R} 99-\mathrm{EJM}$ ). Given the $100 \mathrm{~mm}$ travel range and the \pm 1 $\mu \mathrm{m}$ positional accuracy of the motion stages, this setup can be made to operate using both near-field (NF) and far-field (FF) electrospinning modes [12,13]. The NF setup was used to obtain aligned fiber mats, whereas the FF setup was used to obtain random fiber mats. In order to transition between NF and FF setups, the applied voltage, spinneret distance, and drum speed were adjusted as per the values given in Table 1.

\begin{tabular}{|lcc|}
\hline \multicolumn{3}{|c|}{ Table 1: ElectrospinningConditions } \\
\hline E-Spinning Parameters & Aligned Fiber Mats & Random Fiber Mats \\
\hline $\begin{array}{l}\text { Positive voltage } \\
\text { (Syringe needle) }\end{array}$ & $0.2-1.0 \mathrm{kV}$ & $2.0-3.0 \mathrm{kV}$ \\
Negative voltage (NV) & $1.0-3.0 \mathrm{kV}$ & $1.0-3.0 \mathrm{kV}$ \\
NV Location & Interior pin electrode & Aluminum carrier substrate \\
$\begin{array}{l}\text { Distance between } \\
\text { syringe needle and } \\
\text { drum collector }\end{array}$ & $<1 \mathrm{~cm}$ & $8-10 \mathrm{~cm}$ \\
Drum collector speed & $\sim 5000 \mathrm{rpm}$ & $\sim 10 \mathrm{rpm}$ \\
\hline
\end{tabular}




\subsubsection{Aligned Fiber Mats (Near-field Setup)}

In the NF setup, the aluminum carrier substrate served as the ground electrode while a positive voltage of $0.2-1.0 \mathrm{kV}$ was applied directly to the syringe needle using a high-voltage DC power supply (Gamma High Voltage ES-50). In addition, a negative polarity voltage of 1.0-3.0 kV was also applied to the pin electrode inside the rotating collector drum (Fig. 3c). This configuration of electrodes allowed for the focusing of the electric field lines, which in turn resulted in alignment of the fibers being collected on the aluminum carrier substrate [12]. As with all NF electrospinning processes, this collection method took advantage of the stability region of the polymer jet, which requires the spinneret to be less than $1 \mathrm{~cm}$ from the collector. During the direct-write process, the surface speed of the rotating mandrel ( $\sim 5000$ RPM) was matched tothe speed of the jet.

\subsubsection{Random Fiber Mats (Far-field Setup)}

In the FF setup the pin electrode served as the ground while a positive voltage of 2.0-3.0 kV was applied directly to the syringe needle. In addition, a negative voltage of 1.0-3.0 kV was applied to the surface of the rotating aluminum carrier substrate, which was positioned $\sim 8-10 \mathrm{~cm}$ away from the needle. This electrode arrangement coupled with a slow speed of the drum collector (10 RPM in this case) broadens the electric field lines over a larger area, thereby resulting in the creation of random fiber mats.

\subsubsection{Characterization of the Fiber Mats}

Effective area coverage (EAC) of a fiber mat is defined as the percentage area of the aluminum carrier substrate that is covered by the fibers in a mat. It can be measured by doing a digital image analysis on the scanning electron microscopy (SEM) images of the fiber mats.Since all the fiber mats were made by completing a single pass of the syringe pump along the length of the rotating drum collector, the EAC of the mats was controlled by changing the traversing speed of the needle across the drum collector. EACs in the range of 40-90\% were obtained for both the aligned and the random fiber mats by varying the traversing time of the needle between 6 seconds to 48 minutes. In order to characterize the mats, they were sputter-coated with platinum for 60 seconds and then observed under a Zeiss Supra-55 SEM at an accelerating voltage of $5 \mathrm{kV}$.

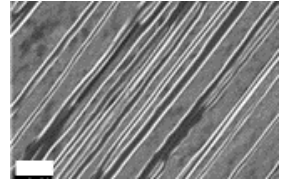

a) EAC $45 \%$

$\boldsymbol{p}=7$ fibers per $100 \mu \mathrm{m}^{2}$

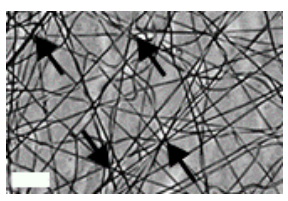

d) EAC $45 \%$, $\boldsymbol{p}=24$ fibers per $100 \mu \mathrm{m}^{2}$

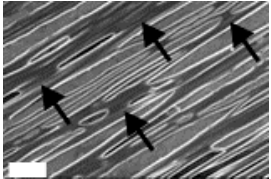

b) EAC $60 \%$ $\boldsymbol{p}=11$ fibers per 100 $\mu \mathrm{m}^{2}$

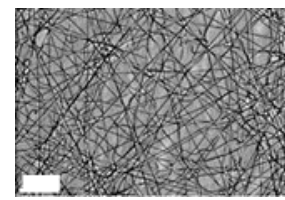

e) EAC $60 \%$

$\mathbf{p}=33$ fibers per $100 \mu \mathrm{m}^{2}$

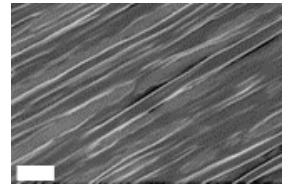

c) EAC $80 \%$

$\boldsymbol{p}=15$ fibers per $100 \mu \mathrm{m}^{2}$

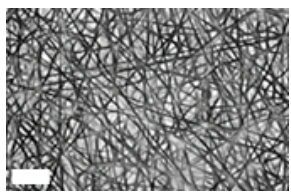

f) EAC $80 \%$

$\mathbf{p}=41$ fibers per

$100 \mu \mathrm{m}^{2}$

Fig. 4: Differences between fiber mats at various effective area coverage (EAC) values ( $\boldsymbol{\beta}$ indicates the fiber number density) $($ Scale bar $=10 \mu \mathrm{m})$

Figures 4a-f depict the characteristic fiber mats obtained for different EAC values. In the case of the aligned fiber mats, while the motion of the stages was expected to translate into the fiber spacing between individual fibers, the stochastic nature of the NF electrospinning process resulted in some fibers being placed close enough to have lateral attachmentwith neighboring fibers(indicated in Fig. 4b by arrows). As the EAC values increased, more of the aligned fibers were seen to have lateral attachment regions (Fig. 4ac). For the random fiber mats, the fibers had overlaying attachment nodes (indicated by arrows in Fig. 4d). The number of these overlaying attachment nodes also increased with an increase in the EAC values (Figs. 4d-f).

The SEM images were also used to compile the fiber diameter distribution profiles by using the ImageJ(C) digital analysis software [15]. The diameter of the aligned fiber mats follows a log-normal distribution with a mean of $1.34 \mu \mathrm{m}$ and a standard deviation of $0.562 \mu \mathrm{m}$ (Fig. 5a). The diameter of the random fiber mats also had a log-normal distribution with a mean and standard deviation of $0.45 \mu \mathrm{m}$ and $0.164 \mu \mathrm{m}$, respectively (Fig. 5b). As seen from the distribution parameters, the diameter of the aligned fiber mats (collected using the NF electrospinning technique) is greater than those of the random fiber mats (collected by the FF electrospinning technique), by a factor of three.

While comparing the aligned and the random fiber mats with the same EAC value, one has to note that the fiber number density (p) of the mat, defined as the number of individual fibers per $100 \mu \mathrm{m}^{2}$ area of the carrier substrate, will be different for both the mats given the difference in the diameter of the fibers. Figures 4a-f list the approximate $\mathbf{p}$ values for each of the six mats depicted. As expected, the aligned fiber mats have a lower fiber number densitycompared to the random fiber mats. 


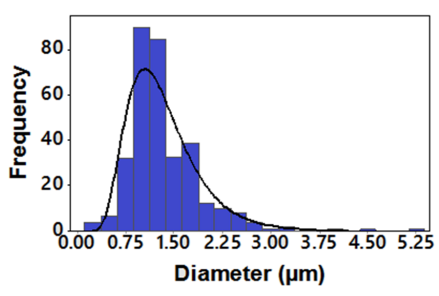

(a)

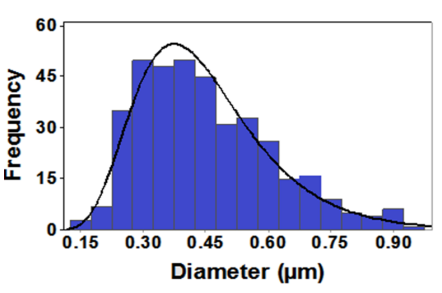

(b)

Fig. 5: Fiber diameter log-normal distributions (a) Aligned fiber mat (b) Random fiber mat

\section{EFFECT OF FIBER CARRIER SUBSTRATE}

The 3D printing process involving the stamping of the fibers onto the polymer layer is inherently a surface energy driven process [16]. In order to enable the choice of appropriate carrier substrates for the 3D printing operation, it is critical to understand the effect of the surface energy of the carrier substrate on the fiber transfer efficiencies encountered during the stamping process.

\subsection{Carrier Substrate Characterization}

In order to study the effect of the surface energy of the carrier substrate, two $100 \mu \mathrm{m}$ thick aluminum films were used in this study, one of which had a coating of polytetrafluoroethylene (PTFE) and the other was uncoated aluminum. The surface energies of these films were characterized using contact angle measurements conducted at 80 degrees (F) and at $24 \%$ relative humidity. Deionized water was dropped from a pressurized syringe onto both of the substrates. The droplets were observed using a high resolution camera (Infiniti ${ }^{\mathrm{TM}}$ ) with a $5 \mathrm{X}$ lens (Fig. 6).For a liquid with a known surface tension value $\left(\gamma_{l}\right)$, the advancing contact angle of the droplet $\left(\theta_{a}\right.$ in Fig. 6) can be used to estimate the total surface free energy $\left(\gamma_{s}^{\text {tot }}\right)$ of the substrate $[17,18]$ by using the relationship:

$$
\gamma_{s}^{\text {tot }}=\frac{\gamma_{l}}{2}\left(1+\cos \left(\theta_{a}\right)\right)
$$

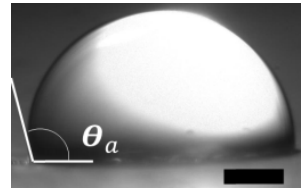

(a)

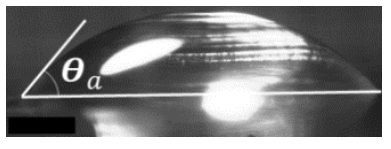

(b)

Fig. 6: Differences in contact angle of DI water on aluminum carrier substrates (a) PTFE coated (b) Uncoated(Scale $=120$ $\mu \mathrm{m})$

As seen from the calculated values from Table 2, the PTFE coated aluminum film had a lower surface free energy than its uncoated counterpart.

\begin{tabular}{|ccc|}
\hline \multicolumn{3}{|c|}{ Table 2: Surface energy of carrier substrate } \\
\hline Material & $\begin{array}{c}\text { Average Contact } \\
\text { Angle (Degrees) }\end{array}$ & $\begin{array}{c}\text { Calculated } \\
\text { Surface Free } \\
\text { Energy (mJ/m } \mathbf{~} \text { ) }\end{array}$ \\
\hline $\begin{array}{l}\text { PTFE-coated } \\
\text { aluminum film }\end{array}$ & 94.1 & 33.4 \\
$\begin{array}{l}\text { Uncoated } \\
\text { aluminum film }\end{array}$ & 39.7 & 63.6 \\
\hline
\end{tabular}

\subsection{Fiber Transfer Efficiency Study}

The 3D printed FrSC parts can have consistent material properties only if the fiber transfer processstays consistent across multiple stamping operations. In order to conduct the fiber transfer efficiency study, fiber mats with effective area coverages in the 40-90\% range were collected on both the PTFE coated and uncoated aluminum carrier substrates. The fiber mat coupons were then mounted on the modified fiber layering system (Figs. 1 \& 2) of the 3D printer. The inkjet-based deposition system was then used to deposit a 15 mm X 15 mm layer of the UV curable base polymer (FullCure 930 TangoPlus $^{\mathrm{TM}}$, Stratasys, Inc.), as per the recipe in Spackman et al. [9]. After the UV curing of the polymer, the fiber mats were cut to the desired size (15 mm X $15 \mathrm{~mm})$ and then stamped onto the polymer layer. For the stamping operation, the modified FLS was first lowered at a velocity of $10 \mathrm{~mm} / \mathrm{min}$ until the high-density elastomer was compressed by $5 \mathrm{~mm}$. The FLS was then raised at a velocity of $10 \mathrm{~mm} / \mathrm{min}$ until the fiber carrier substrate was inclined 
at its maximum angle or $30^{\circ}$ (Fig.2). Beyond this height, the FLS was cleared away from the substrate by moving at a rate of 100 $\mathrm{mm} / \mathrm{min}$. The stamping studies were conducted at a room temperature of $20{ }^{\circ} \mathrm{C}$ and a relative humidity of $70 \%$.

In an ideal scenario, $100 \%$ of the fibers from the intended area would betransferred from the carrier substrate to the 3D printed part. However, depending on the nature of the mat (alignment, effective area coverage, and diameter of the fibers) and the relative value of the surface energy between the fibers and the UV cured polymer, all the fibers may not bereleased from the carrier substrate. Figure 7 depicts one such scenario involving a PTFE coated aluminum substrate and an aligned fiber mat that indicates a partial release of the fibers during the stamping operation. The edges of the image correspond to the $15 \mathrm{~mm} \mathrm{X} 15 \mathrm{~mm}$ releasing cut made by the tungsten carbide cutter that aims to release fibers from that area. However the fibers are only released in the region A-B-C-D-E-F (Fig. 7). The fiber transfer efficiency (FTE) in this case is defined as

$$
\text { FTE }=100 * \frac{\text { Effective area of transferred fibers }}{\text { Intended area of fiber transfer }} .
$$

In Fig. 7, the numerator of Eq. 2 is the area of the region A-B-C-D-E-F, whereas the denominator is the $15 \mathrm{X} 15 \mathrm{~mm}$ area that was scribed using the tungsten carbide cutter.

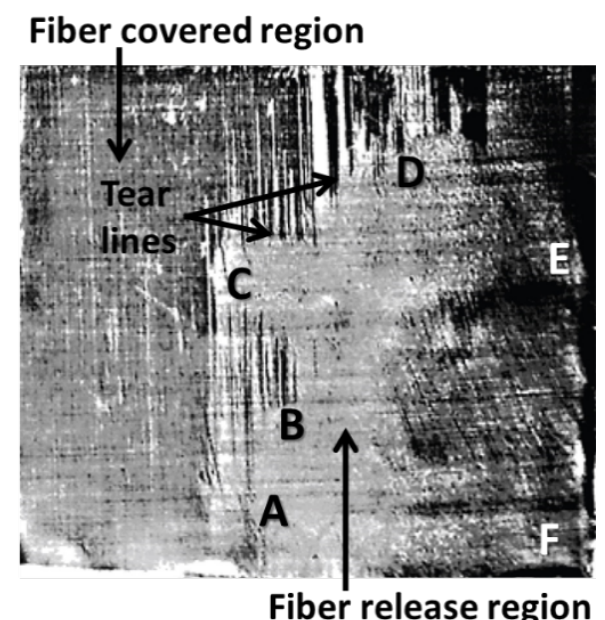

Fig. 7: Example of partially transferred mat (FTE 60\% (Note: Tear lines indicate mat failure)

Figure 8 depicts the FTE plotted as a function of the EAC for both the aligned and the random fiber mats collected on the coated and uncoated aluminum substrates. There exists a single curve for the uncoated aluminum carrier substrate since no differences were observed between the performance of the aligned and the random fiber mats. While interpreting this graph, it should be noted that due to the difference in the fiber diameter distributions (Fig. 5a-b)though both the fiber mats may have the same EAC,theirfiber number density ( $(\boldsymbol{\partial})$ will be different.

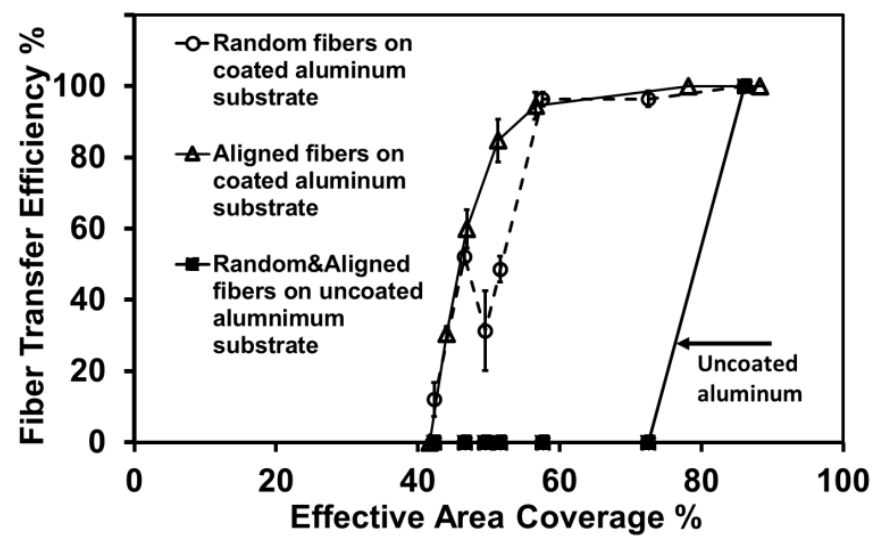

Fig. 8: Fiber transfer efficiency curves

As seen in Fig. 8, the uncoated substrate had no transfer of fibers until an EAC of 80\%, and at EACs $\geqq 80 \%$, the FTE was nearly $100 \%$. At EACs $<40 \%$ both the PTFE coated and uncoated substrates wereunable to transfer any of the fibers. However, at EACs between $40-80 \%$, the PTFE coated aluminum substrate was seen to perform better than the uncoated carrier substrate, for both typesof the fiber mats. 
For aligned fiber mats collected on the PTFE coated carrier substrate, one observes an almost linear increase in the FTE with an increase in the EAC in the 40-80\% range. There are two probable reasons for this. First, the aligned fibers have a larger diameter than the random fibers which enables them to withstand the peeling load experienced during the stamping operation, without tearing the mat. Second, with an increase in the EAC of an aligned fiber mat, there is a proportionate increase in the number of lateral attachment regions between the fibers (Fig. 4b), which further increases the probability of fiber transfer. Both these factors coupled with the lower number density of the aligned fiber mats are responsible for the increase in the FTE, with an increase in the EAC for the aligned fiber mats.

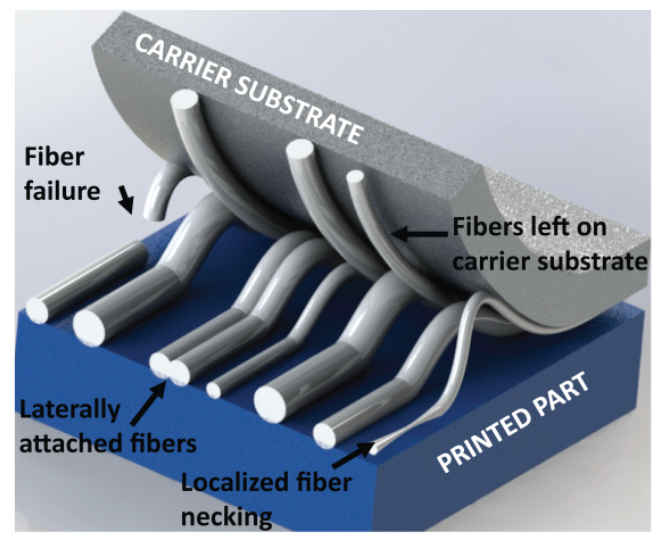

(a)

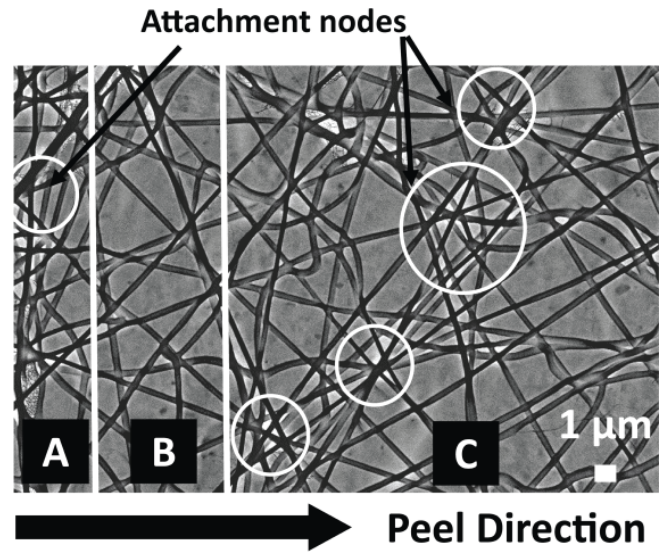

(b)

Fig. 9: Mechanisms responsible for fiber transfer efficiency trends (a) Aligned mat (b) Random mat

Figure 9a depicts some of the likely scenarios encountered while stamping aligned fiber mats at EACs in the $40-80 \%$ range. As seen, a majority of the fibers are transferred to the printed part due to the larger diameter of the fibers and the lateral attachment between fibers. A fraction of the mat remains on the carrier substrate while some fibers fail under the peeling load due to localizednecking (caused by stress concentrators such as beads formed under electrospinning conditions). The breaking of the fibers appears in the form of tear lines that are visible on the mat (refer Fig. 7).

In general, the random fiber mats show an increasing trend in FTE with an increase in the EAC values, except for the EAC values in the range of $45-55 \%$ where the FTE stays more or less constant at $\sim 50 \%$. The fibers, in the case of random fiber mats,are smaller in diameter compared to the aligned fibers, and therefore more prone to failure under the stress generated by the peeling load. Thus, an improvement in the FTE of a random mat is likely correlated to the presence of overlaying fiber attachment nodes that result in an increased adhesion between the mat and the polymer layer.

Figure $9 \mathrm{~b}$ shows a random fiber mat with an EAC of $~ 50 \%$. Regions A and $\mathrm{C}$ of this mat have a greater number of the overlaying fiber attachment nodes (refer Fig. 4d indicated by black arrows). Region B lies between these two relatively strong sections of the fiber mat, and has fewer attachment nodes. Therefore, as the peel front propagates through Region B, it is likely for a tear to propagate across the mat. As the EAC of the random fiber mat increases these attachment nodes become more common across the mat, and the distance between them shrinks (Fig. 4d-f). This is suspected to be the mat strengthening mechanism responsible for the increase in FTE. For random fiber mats with EACs in the $45 \%-55 \%$ range, the fact that the FTE did not improve much could indicate that the relative percentage of attachment nodes was more or less the same across those mats. 


\section{TENSILE TESTING RESULTS}

In order to characterize the material properties and the failure mechanisms encountered in 3D printed FrSCs, a series of dog-bone specimens were printed with varying fiber loadings. The results from the FTE study showed that the PTFE-coated aluminum carrier substrate was best-suited to ensure the transfer of the fibers during the stamping process. Therefore, it was the only fiber carrier substrate used to 3D print the tensile testing samples.

The overall shape and dimensions of the 3D printed dog-bone FrSC specimens are shown in Fig. 10. The manufacturing process outlined in Section 2 was used to alternately print UV curable ink (FullCure 930 TangoPlus ${ }^{\mathrm{TM}}$, Stratasys, Inc) and to stamp the fiber mats. The UV curable polymer ink was deposited using a $120 \mu \mathrm{m}$ diameter inkjet nozzle. Before the fiber-stamping step, the ink was cured using a UV light source (Dynamax ${ }^{\mathrm{TM}}$ BlueWave 200) with an intensity of $\sim 30 \mathrm{~mW} / \mathrm{cm}^{2}$. For the aligned fiber mats, the fibers were stamped such that the direction of alignment coincided with the Y-axis of the 3D printer (Fig. 10).

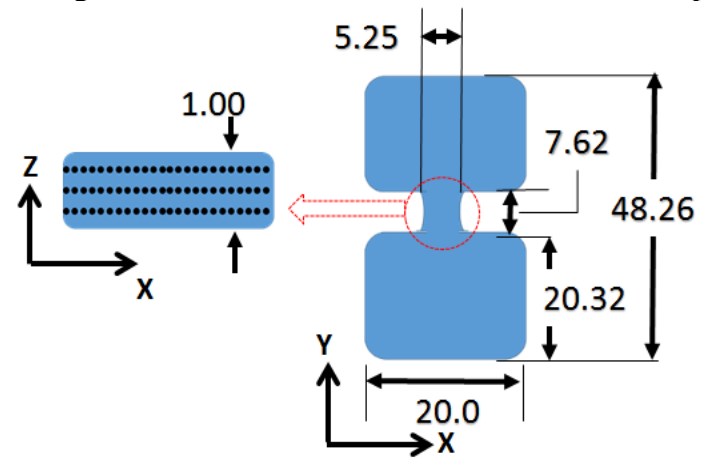

Fig. 10: Tensile testing sample (Note: All distances are in millimeters, figure is not to scale)

The aligned and the random composite samples were printed with three levels of fiber loading. The volume fractions corresponding to the low, medium and high levels of fiber loading are estimated in Table 3 as:

$$
\text { Estimated Vol. Fraction }(\%)=\frac{\text { Fiber mat thickness }}{\text { Printed polymer layer thickness }} * \mathrm{FTE} * \mathrm{EAC} * 100 \text {, }
$$

With the thickness of the printed layer being $~ 75 \mu \mathrm{m}$ for all the composites, the low, medium, and high levels of fiber loading correspond to volume fractions of $0.16 \%, 1.08 \%$, and $1.65 \%$, respectively, for the random fiber composites.

The corresponding volume fractions for the aligned composites were estimated to be $0.14 \%, 0.68 \%$, and $1.6 \%$, respectively, for the low, medium, and high levels of fiber loadings. These low levels of fiber volumes are in-line with the findings of the thermogravimetric tests conducted by Spackman et al. [9]. Plain polymer specimens were also printed and tested to generate the baseline data. To characterize the properties of the fiber mats, both aligned and random fiber mats (10 $\mu \mathrm{m}$ thick) were also tested as per the protocols laid out in Huang et al. [19].

Custom clamps were used to secure the 3D printed tensile dog-bone specimens at both ends. An average strain rate of $0.065 s^{-1}$ was applied to all the samples. Three samples of each of the six fiber composites, neat polymer, and two fiber mat types were destructively tested. Figure 11a depicts the progressive failure seen in a sample of the random fiber mat tested, whereas Fig. 11b shows representative curves for all of the materials tested. It should be noted that in Fig. 11b the vertical axis for the mats is on the right (indicated by *), whereas the axis for the composite and the plain polymer is on the left.

The mechanical properties obtained for all the composites are compiled in Table 3. In general, the elastic modulus of the aligned composite is seen to be higher than that of the random composite, except for the medium-level of fiber loading where it is lower than that for the random composite. However, this trend makes sense when the numerical values of the fiber fraction estimates are compared. At the medium-level of fiber loading the aligned composite has only $0.68 \%$ volume fraction of fibers, whereas the random composite has a higher percentage of $1.08 \%$.The results reveal that the aligned fiber mat has a higher modulus and ultimate tensile stress than the random fiber mat. The elastic modulus of both the random and the aligned composites is seen to be higher than that of the plain polymer. 

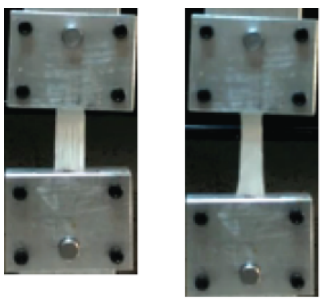

$\mathbf{t}=\mathbf{0}$

$t=30 s$

Axis for

3D printed material

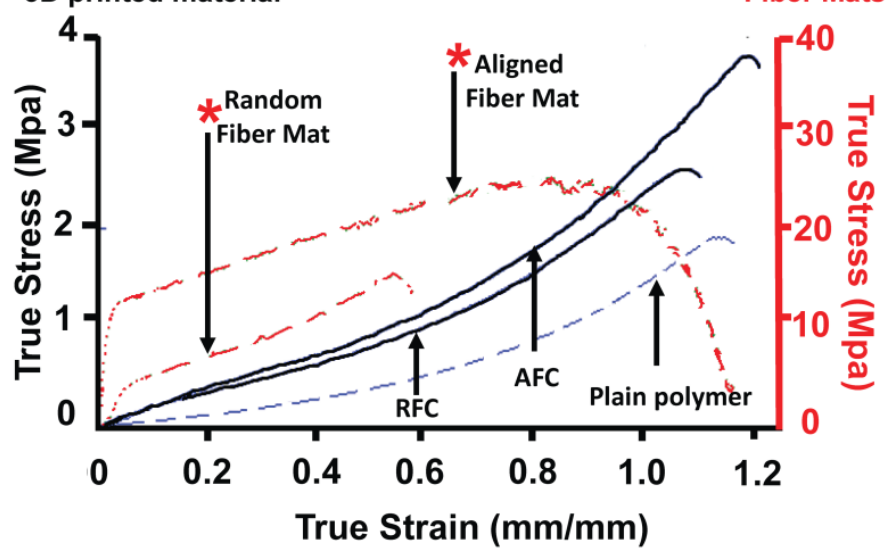

(b)

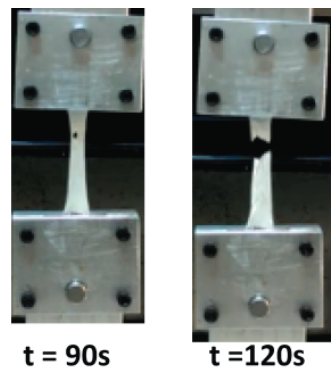

(a)
* Axis for Fiber Mats

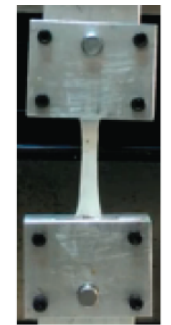

$t=90 s$

$$
\text { . }
$$




\section{DEFORMATION AND FAILURE MECHANISMS}

\subsection{Microscopy Evidence}

The cross-sections of the fractured dog-bone samples were imaged using a SEM to study the various deformation and failure mechanisms responsible for the improved mechanical properties seen in 3D printed FrSCs (Figs. 12a-f). These findings include the following:

- Local plastic deformation (Crazing): The aligned nylon fibers in the polymer matrix are seen to act as stress concentrators that result in local plastic deformations similar to crazing [20] (Fig. 12a). The formation of these craze lines is expected to serve in dissipating the fracture energy during failure.

- Crack arrest: Figure 12b depicts an example of a layer of fibers arresting the propagation of a crack front. Such a crack arrest mechanism is absent in the 3D printed plain polymer samples.

- Fiber-reinforcement: While FrSCs inherently comprise of two polymer systems (fiber+matrix) coming together to form the composite, the fibers are seen to act as reinforcement agents bridging across crack faces (Fig. 12c). This mechanism seen for the 3D printed composites is comparable to the fiber-reinforcement of cracks observed in carbon nanotube-reinforced polymer composites [21]

- Crack Deflection: In addition to crack arrest (Fig. 12b) the presence of the fiber mats is seen to result in crack deflection across multiple layers. As seen in Fig. 12d, the crack front, after being arrested along path AB (by Fiber Layer 1), is seen to propagate along path BC and is then deflected along path CD (by Fiber Layer 2).

- Fiber pull-out: The classic fiber pull-out scenario is also encountered in these composites (Figs. 12 e,f). Figure 12e shows the fiber pull-out holes left behind in the composite, whereas Fig. $12 \mathrm{f}$ shows evidence of the pull-out ends of the fibers. It can be seen that the diameter of the holes left behind by the fiber pull-out are larger than the diameter of the fibers themselves, with some holes being $\sim 5$ microns (Fig. 12e). This could be explained by the likely formation of a polymer sheath around the fibers [22], which is indicative of a good bonding between the fiber reinforcements and the polymer matrix.

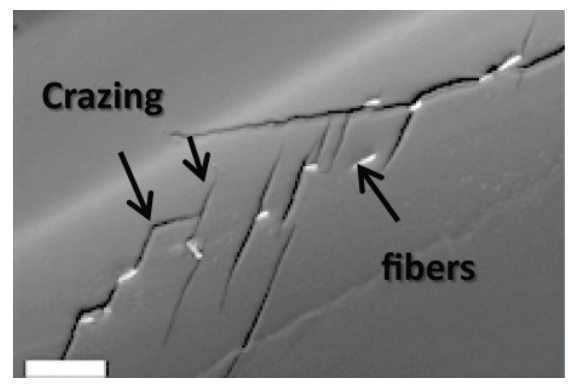

(a) Local plastic deformation/Crazing

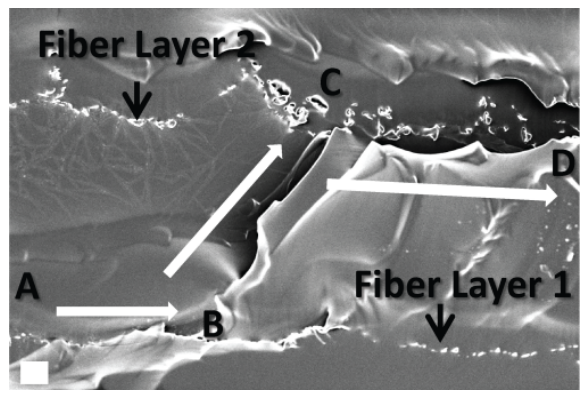

(d) Crack deflection by fiber mats

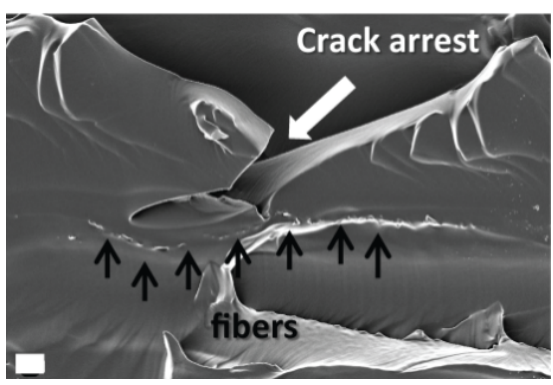

(b) Crack arrest by fiber mat

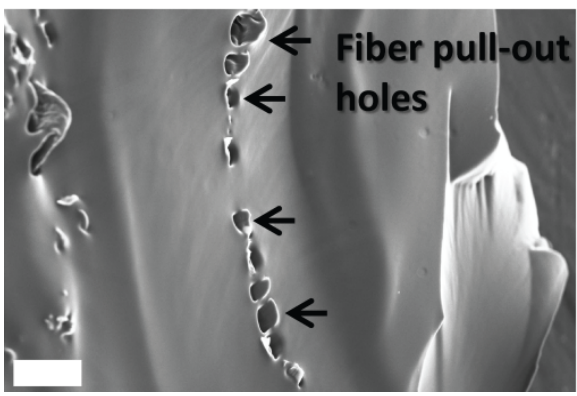

(e) Fiber pull-out holes

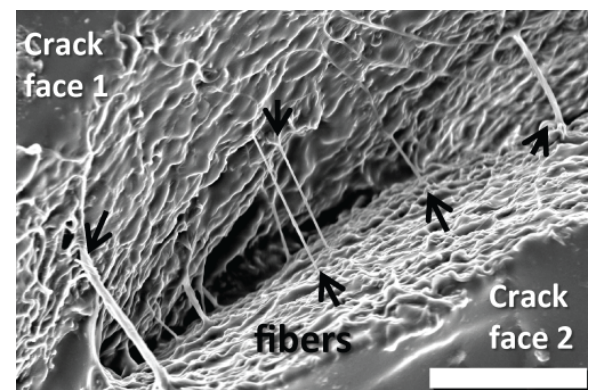

(c) Fiber-reinforcement of cracks

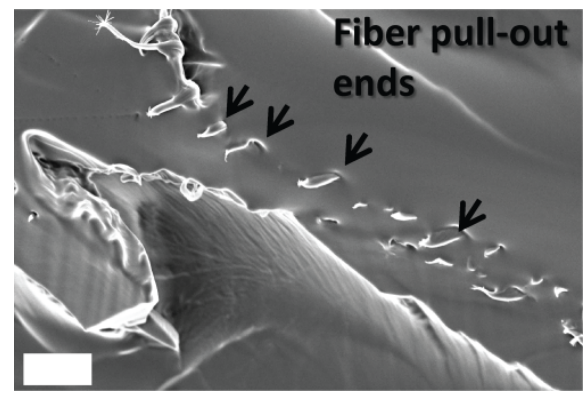

(f) Fiber pull-out ends

Fig. 12: Deformation mechanisms seen in 3D printed fiber-reinforced soft composites (Scale $=10 \mu \mathrm{m})$

\subsection{Estimating the Elastic Modulus of the Interfacial Polymer Sheath}

The two-phase rule of mixtures for the elastic modulus $\left(\mathrm{E}_{\text {comp . }}^{2-\text { phase }}\right)$ of the 3D printed FrSCs, is given by

$$
\mathrm{E}_{\text {comp }}^{2-\text { phase }}=\mathrm{E}_{\mathrm{m}}\left(1-\mathrm{V}_{\mathrm{f}-\mathrm{mat}}\right)+\mathrm{E}_{\mathrm{f}-\mathrm{mat}} \mathrm{V}_{\mathrm{f}-\mathrm{mat}} \text {, }
$$


where, $V_{f-m a t}$ and $E_{f-m a t}$ are the volume fraction and the elastic modulus of the fiber mat, respectively, andE $E_{m}$ is the elastic modulus of the polymer matrix [22]. Figure 13 plots a comparison between the experimental data obtained for the elastic modulus of the composites and the values predicted by the two-phase rule of mixtures presented in Eq. 4. The results in Fig. 13 show that the twophase model always under-predicts the elastic modulus of the FrSCs.

In the case of the FrSCs, it has been observed that the polymer matrix surrounding the fibers has different material properties as opposed to the bulk polymer [22,23]. The three-phase rule of mixtures for the elastic modulus ( $\mathrm{E}_{\text {comp }}^{3-\mathrm{phase}}$ ) of the 3D printed FrSCs accounts for the elastic modulus of this interfacial polymer sheath and is given by:

$$
\mathrm{E}_{\text {comp. }}^{3-\text { phase }}=\mathrm{E}_{\mathrm{m}}\left(1-\mathrm{V}_{\mathrm{f}-\mathrm{mat}}-\mathrm{V}_{\mathrm{i}}\right)+\mathrm{E}_{\mathrm{f}-\mathrm{mat}} \mathrm{V}_{\mathrm{f}-\mathrm{mat}}+\mathrm{E}_{\mathrm{i}} \mathrm{V}_{\mathrm{i}} \text {, [Eq. 5] }
$$

where the additional interfacial terms $E_{i}$ and $V_{i}$ are the elastic modulus and volume fraction of the interfacial polymer sheath, respectively. If the $V_{i}$ values can be estimated for the FrSCs then the elastic modulus of the interfacial polymer sheath can be estimated by fitting Eq. 5 to the data listed in Table 3.

The volume fraction, $\mathrm{V}_{\mathrm{i}}$, of the interphase can be estimated as:

$$
\mathrm{V}_{\mathrm{i}}=[\mathrm{C}-1] * \mathrm{~V}_{\mathrm{f}-\mathrm{mat}}
$$

whereC is given by:

$$
C=\frac{\pi \bar{r}_{\text {hole }}^{2}}{\pi \bar{r}_{\text {fiber }}^{2}}=\left(\frac{\bar{r}_{\text {hole }}}{\bar{r}_{\text {fiber }}}\right)^{2}
$$

In Eq. $7 \pi \bar{r}_{\text {hole }}^{2}$ is the average cross-sectional area of the fiber pull-out holes and $\pi \overline{\mathrm{r}}_{\text {fiber }}^{2}$ is the average cross-sectional area of the fibers in that specimen (according to the distribution curve in Fig. 5). Fiber pull-out images similar to Fig. 12e were analyzed for all the six types of FrSCs listed in Table 3. The cross-sectional area of the fiber pull-out holes was used to first calculate C (Eq. 7) and then to calculate $V_{i}$ (Eq. 6). These calculations yield the estimated $V_{i}$ values to be $0.78 \%, 5.3 \%$ and $8.2 \%$ for the random fiber composite with low, medium and high fiber loadings, respectively (Table 3). The estimated $V_{i}$ values for low, medium and high fiber loadings for the aligned composite are $0.68 \%, 3.4 \%$, and $8 \%$, respectively. The elastic modulus of the interfacial polymer sheath was then estimated by fitting Eq. 5 to the data listed in Table 3. For this class of FrSCs the value of $\mathrm{E}_{\mathrm{i}}$ lies in the range of 1.75-1.95 MPa, which is one order of magnitude higher than the base polymer and an order of magnitude lower than that of the fiber mats.

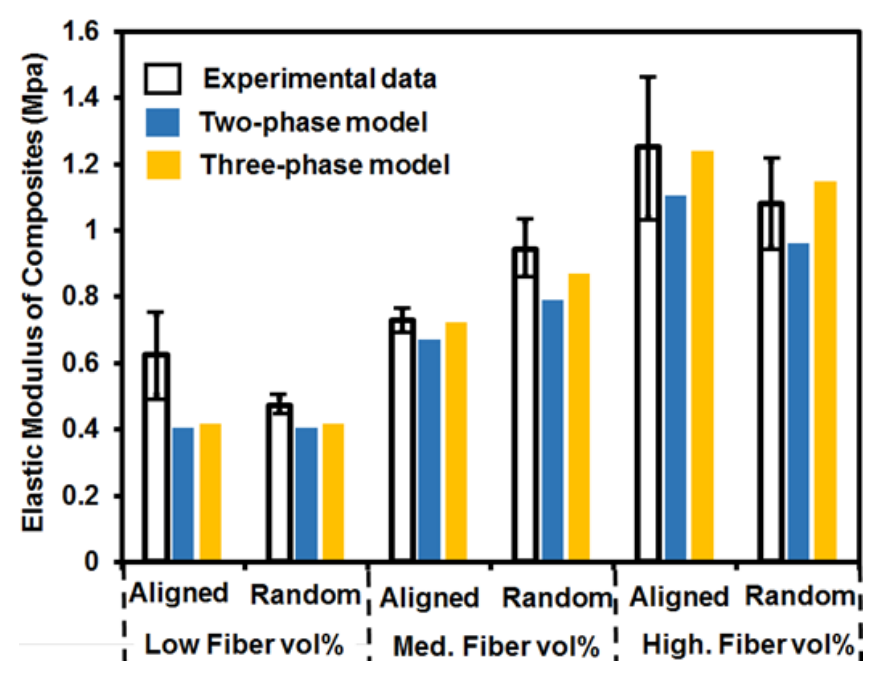

Fig. 13:Rule of mixtures prediction of FrSC elastic modulus values

Figure 13 shows the predictions made by the two-phase and the three-phase rule of mixtures when compared to the experimental data. For both the aligned and the random fiber composites, the three-phase model is seen to perform better than the two-phase model, especially at the medium and high fiber volume percentages. With an increase in fiber loadings, one expects a greater dominance of the interfacial polymer sheath in dictating the properties of the composites. This would explain the superior performance of the threephase model at the medium and high fiber volume percent. At the low volume fiber loading, both the two-phase and the three-phase 
model predictions were seen to be comparable, and both these models consistently under predict the elastic modulus of the composite. This is likely due to the possible error in the fiber volume estimates, especially at the low percentage values.

\section{CONCLUSIONS}

In this paper the 3D printing technique for fiber-reinforced soft composites developed by Spackman et al. [9] was improved using three modifications, viz., 1) The design of an electrospinning-based "direct-write" system for polymer fiber coupons; 2) Use of carrier substrates with varying surface energies; and 3) A modified fiber layering system that allows for a peeling action of the fibers during the stamping operation. The key findings from this study include the following:

1. The direct-write system relies on the use of a near-field electrospinning system with a custom-designed drum collector that allows for varying fiber carrier substrates to be mounted. The precision motion platform allows for the manufacture of fiber mats of varying area coverage and number densities. Both the aligned and the random fiber mats are seen to have a log-normal distribution with the average diameter of the fibers being $1.5 \mu \mathrm{m}$ and $0.45 \mu \mathrm{m}$ for the aligned and the random fiber mats, respectively.

2. The surface energy of the carrier substrate influences the fiber transfer efficiency of the stamping operation used during the 3D printing process. PTFE coated aluminum substrates are seen to outperform uncoated aluminum carrier substrates, especially for fiber mats having effective area coverage in the $40-80 \%$ range. The diameter of the fibers and the nature of the attachment points between the fibers are both seen to influence the fiber transfer efficiency.

3. Tensile testing of the mats shows that the aligned fiber mat has a higher elastic modulus and ultimate tensile stress than the random fiber mat.

4. The tensile tests of the 3D printed FrSCs show that the addition of the nylon fibers improves the elastic modulus of the composite by $40 \%-260 \%$, depending on the alignment and the fiber content in the composite. The aligned composites are generally seen to have a higher Young's modulus than both the base polymer and the random composites.

5. The 3D printed FrSCs show evidence of characteristic failure mechanisms seen also in the domain of nanocomposite materials. These include fiber-induced local plastic deformation (crazing), crack arrest and deflection, fiber strengthening, and fiber pullout.

6. The evidence of fiber pull-out also points to the presence of an interfacial polymer sheath around the fibers. The elastic modulus of this interfacial polymer is estimated to be an order of magnitude higher than the base polymer.

\section{ACKNOWLEDGEMENT}

The authors gratefully acknowledge funding support from the US National Science Foundation (Award Number CMMI 14-62648)

\section{REFERENCES}

[1] Tibbits, S., 2014, "4D Printing: Multi-material Shape Change", Architectural Design, 84(1), pp. 116-121.

[2] Ge, Q., Qi, H.J., and Dunn, M.L., 2013, “Active Materials by Four-dimension Printing”, Applied Physics Letters, 103(13), 131901 (5 pp.).

[3] Lapidot, S., Meirovitch, S., Sharon. S., Heyman A., Kaplan DL., and Shoseyov, O., 2012, "Clues for Biomimetics from Natural Composite Materials",Nanomedicine, 7(9), pp. 1409-1423.

[4] Yan, F., Liu, Y., Chen, H., Zhang F., Zheng, L., and Hu, Q., 2014, “A Multi-scale Controlled Tissue Engineering Scaffold Prepared by 3D Printing and NFES Technology”, AIP Advances, 4 (3), 031321 (8 pages).

[5] Czyzewski, J., Burzynski, P., Gawe, K., and Meisner, J., 2009, "Rapid Prototyping of Electrically Conductive Components Using 3D Printing Technology”, Journal of Materials Processing Technology, 209(12-13), pp. 5281-5285.

[6] Rocha,C.R., Perez, T., Angel, R., Roberson, D.A., Shemelya, C.M., MacDonald, E.W., and Wicker, R.B., 2014, "Novel ABSbased Binary and Ternary Polymer Blends for Material Extrusion 3D Printing", Journal of Materials Research, 29(17), pp. 18591866.

[7] Lopes, A.J., MacDonald,E., Wicker, R.B., 2012, "Integrating stereolithography and direct print technologies for 3D structural electronics fabrication", Rapid Prototyping Journal, 18(2), pp. 129-143.

[8] Wang L., Ma Y., Chen M., Yao H., Zheng X., and Yang W., 2010, "An Inkjet Printing Soft Photomask and its Application on Organic Polymer Substrates", Science China: Chemistry, 53(8), pp. 1695-1704.

[9] Spackman, C., Picha, K., Gross, G., Nowak, J., Smith, P.J., Zheng, J., Samuel, J. , and Mishra, S., 2015, “A Novel Multi-material Additive Manufacturing Technique for Fabricating Laminated Polymer Nancomposite Structures”, ASME Journal of Micro and Nano Manufacturing, 3(1), 011008 (11 pages) 
[10] Chronakis, I. S., 2005, “Novel Nanocomposites and Nanoceramics Based on Polymer Nanofibers Using Electrospinning Process-A Review,” Journal of Material Processing Technology, 167(2-3), pp. 283-293.

[11] Carter, W., Popell, G.C., Samuel, J., and Mishra, S., 2014, "Fundamental Study and Modeling of the Droplet Formation in Nearfield Electrohydrodynamic Printing”, ASME Journal of Micro and Nano Manufacturing , 2(2), 021005 (12 pages)

[12] Chang, C., Limkrailassiri1, K., and Lin, L., 2008, “Continuous near-field electrospinning for large area deposition of orderly nanofiber patterns”,Applied Physics Letters 93, 123111 (4 pages).

[13] Teo, W. E., and Ramakrishna, S., 2006, “A Review on Electrospinning Design and Nanofibre Assemblies”, Nanotechnology, 17(14), pp. 89-106.

[14]Bazbouz, M. B., and Sylios, G. K., 2008, “Alignment and Optimization of Nylon 6 Nanofibers by Electrospinning”, Journal ofAppied. Polymer Science, 107(5), pp. 3023-3032.

[15] Schneider, C.A., Rasband, W.S., and Eliceiri, K.W., 2012, "NIH Image to ImageJ: 25 years of image analysis", Nature Methods, 9, pp. 671-675.

[16] Duan, Y., Huang,Y., Yin, Z., 2013, "Transfer printing and patterning of stretchable electrospin film", Thin Solid Films, 544, pp. 152-156.

[17] Chibowski, E. 2003. "Surface free energy of a solid from contact angle hysteresis", Advances in Colloid and Interface Science, 103, pp. 149-172.

[18] Chibowski, E., 2007, “On some relations between advancing, receding and Young's contact angles”, Advances in Colloid and Interface Science, 133,pp. 51-59

[19]Huang, Z.M., Zhang, Y.Z., Ramakrishna, S., Lim, C.T.., 2004, "Electrospinning and mechanical characterization of gelatin nanofibers”, Polymer, 45, pp. 5361-5368.

[20]Zhang,W., Srivastava, I., Zhu, Y., Picu, C.R., Koratkar, N., 2009, "Heterogeneity in epoxy nanocomposites initiates crazing: significant improvements in fatigue resistance and toughening", Small, 5(12), pp. 1403-7.

[21] Suhr,J., Koratkar, N., Keblinski, P., and Ajayan, P., 2005, "Viscoelasticity in carbon nanotube composites", Nature Materials, (4), pp. 134-137.

[22] Coleman, J.N., Khana, U., Blaua, W.J., Gun’kob Y.K., 2006, "Small but strong: A review of the mechanical properties of carbon nanotube-polymer composites”, Carbon, 44, pp. 1624-1652.

[23] Herrera-Franco, P.J.,Valadez-Gonzalez, A., 2004, "Mechanical properties of continuous natural fibre-reinforced polymer composites”, Composites: Part A, 35, pp. 339-345. 


\section{LIST OF FIGURES}

Fig. 1: 3D printing platform for fiber-reinforced soft composites ( $a$ - inkjet nozzles, $b$ - fiber layering system, $c$ - UV cure lamp, $d$ $U V$ curing rod lens, $e$ - heat lamp, $f$ - pneumatic slides, $g$ - printing substrate, $h$ - tungsten carbide cutter) [9]

Fig. 2: Overall manufacturing process cycle for 3D printing FrSCs

Fig. 3: Direct-write setup for manufacturing polymer fiber mats (a) Overall set-up configuration (b) Close-up of the interior of the drum collector (c) Near-field configuration for aligned fiber mats (d) Far-field configuration for random fiber mats

Fig. 4: Differences between fiber mats at various effective area coverage (EAC) values ( $p$ indicates the fiber number density) (Scale bar $=10 \mu \mathrm{m})$

Fig. 5: Fiber diameter log-normal distributions (a) Aligned fiber mat (b) Random fiber mat

Fig. 6: Differences in contact angle of DI water on aluminum carrier substrates (a) PTFE coated (b) Uncoated (Scale= $120 \mu \mathrm{m}$ )

Fig. 7: Example of partially transferred mat (FTE 60\% (Note: Tear lines indicate mat failure)

Fig. 8: Fiber transfer efficiency curves

Fig. 9: Mechanisms responsible for fiber transfer efficiency trends (a) Aligned mat (b) Random mat

Fig. 10: Tensile testing sample (Note: All distances are in millimeters, figure is not to scale)

Fig. 11: Tensile testing results (a) Progressive failure seen in the random fiber mat (b) Representative curves (Note: RFC-Random fiber composite and AFC-Aligned fiber composite)

Fig. 12: Deformation mechanisms seen in 3D printed fiber-reinforced soft composites (Scale $=10 \mu \mathrm{m}$ )

Fig. 13: Rule of mixtures prediction of FrSC elastic modulus values 